\title{
Industrial and Systems Safety and Reliability in the Big-Data Era
}

\author{
Min Xie \\ City University of Hong Kong \\ China \\ minxie@cityu.edu.hk
}

\begin{abstract}
Artificial intelligence has become a popular topic and it has been introduced to many industrial systems. Traditional mechanical and manufacturing systems have seen a high level of automation through the adaptation of AI and machine learning. In this talk, we will discuss the issues and challenges in the changing world. With the plenty amount of data collected from various sensors, predictive analytics has helped to ensure system safety and reliability. Although in the field such as reliability engineering, prognostics and predictive maintenance approaches have been enhanced with sensor data and AI methods, systems have an increased complexity and new problems and issues are arising. We will share some of the experience from our research in system engineering and future challenges for researchers and practitioners.
\end{abstract}

